\section{Kidney \\ Blood Pressure \\ Research}

\title{
Evaluation of Renal Function Under Controlled Hypotension in Zero Ischemia Robotic Assisted Partial Nephrectomy
}

\author{
Ester Forastiere ${ }^{a} \quad$ Claudia Claronia ${ }^{a}$ Maria Sofra ${ }^{a} \quad$ Giulia Torregiani $^{a}$ \\ Marco Covotta ${ }^{a}$ Maria Grazia Marchione ${ }^{a}$ Diana Giannarellib Rocco Papaliac

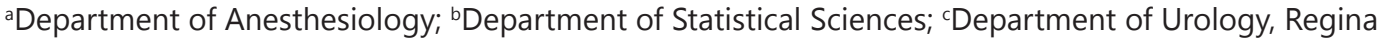 \\ Elena National Cancer Institute, Rome, Italy
}

\section{Key Words}

Robotic partial nephrectomy Renal function - Hypotensive anaesthesia - Zero ischemia • Kidney neoplasms $• 99 \mathrm{mTc}-$ Mercaptoacetyltriglycine

\begin{abstract}
:
Background/Aims: In partial nephrectomy with hilar clamping every minute of ischemia can impair renal function, thus great importance is having the controlled hypotension as a part of zero ischemia technique. The aim of the study is to evaluate the effects of hypotensive anesthesia on renal function, in patients undergoing robotic assisted partial nephrectomy (RAPN), during surgery and at 3 months follow up. Methods: This is a prospective study of 100 patients, ASA 1-2, who underwent zero ischemia RAPN under controlled hypotension $(\mathrm{CH})$ from December 2011 through to May 2013. Serum creatinine, BUN, estimated glomerular filtration rates (eGFR), fractional excretion of sodium (FSE) and technetium Tc $99 \mathrm{~m}$ mercaptoacetyltriglycine (99mTC-MAG-3), renal scintigraphy with effective renal plasma flow (ERPF) were evaluated. Results: Mean duration of $\mathrm{CH}$ was $50 \pm 4$ minutes. Acute renal failure wasn't observed in any of the patients. A significant variation of eGFR during the procedure and 24 hours after surgery was observed. No significant variation of BUN and FSE was detected. Comparing preoperative ERPF of the operated kidney with ERPF 3 months after surgery, it decreased by $2 \%$. Conclusion: In patients with normal preoperative renal function $\mathrm{CH}$ didn't show any detrimental impact on renal function during and after robotic assisted partial nephrectomy.
\end{abstract}

Copyright $(2014$ S. Karger AG, Basel

\section{Introduction}

Elective partial nephrectomy (PN) is considered the gold standard treatment for renal tumors $\leq 7 \mathrm{~cm}$ [1]. Most surgeons use vascular clamping to perform PN. The most common 


\section{Kidney \\ Blood Pressure Research}

Forastiere/Claroni/Sofra et al.: Renal Function After Controlled Hypotension

method used to perform PN is clamping of the renal artery with or without the renal vein (warm ischemia). Since warm ischemia time (WIT) was identified as the most important surgery-related factor affecting renal function in patients undergoing PN, zero ischemia techniques are emerging as a safe and effective approach in tertiary referral centers. [2-5]. We recently reported a series of 60 patients who underwent laparoscopic and robotic PN without hilar clamping and with controlled hypotension during tumor excision, all procedures were completed safely with encouraging outcomes [4]. In this study, we investigated renal function during RAPN under $\mathrm{CH}$ lasting from the beginning of the procedure until the complete repair of renal defect.

\section{Materials and Methods}

We prospectively enrolled 100 ASA 1-2 patients with peripheral renal tumors who underwent zero ischemia minimally invasive partial nephrectomy (MIPN) under CH between December 2011 and May 2013. All patients signed a written informed consent approved by the ethics committee at our center. Abdominal computed tomography (CT) scan and technetium Tc 99m mercaptoacetyltriglycine (99mTC-MAG-3) renal scintigraphy were performed preoperatively in every patient. Inclusion criteria comprised all ASA12 patients with no hilar renal masses considered eligible for RPN regardless of tumor size or pattern of growth, with preoperative normal renal function. Patients with ASA score $>2$, hilar tumors and preoperative CKD (eGFR $<60 \mathrm{ml} / \mathrm{min} / 1.73 \mathrm{~m} 2$ ) were excluded from the study. A transperitoneal approach was performed in all cases. Once accessed to the retroperitoneal space, the Gerota's capsule was opened and the kidney was completely freed and mobilized in order to allow circumferential exposure of the tumor.

Laparoscopic ultrasound was performed to identify endophytic tumors and to establish a safe resection area. The renal parenchyma incision was made just few $\mathrm{mm}$ below the renal tumor and the surgical dissection plane was established. During dissection specific vascular branches supplying the tumor were identified and clipped using Hemo-olock R. Tumor excision was continued and completed with cold scissors using electrocautery when required.

For each patient demographic, operative, perioperative data and postoperative complications classified according to the Clavien system were

recorded and maintained prospectively in a database. Serum creatinine, BUN, eGFR according to the CKD-EPI formula [6], fractional excretion of sodium (FSE) according to the equation: $\mathrm{EFNa} \%$ : [Na]U X [Cr] $\mathrm{P} /[\mathrm{Na}] \mathrm{P} \mathrm{X}$ [Cr]U were measured before hypotensive anesthesia (T0), 40 minutes after HA (T1), 40 minutes after the end of the procedure (T2), 24 hours after surgery (T3) and 3 months after surgery (T4). Technetium Tc 99m mercaptoacetyltriglycine (99mTC-MAG-3) renal scintigraphy was performed preoperatively and 3 months postoperatively.

\section{Hypotensive anesthesia}

Thirty minutes before induction of anesthesia, all patients received $10 \mathrm{mg}$ of

intravenous ketoralac trometamina or $100 \mathrm{mg}$ of tramadolo cloridrato, $100 \mathrm{mg}$ of ranitidine, and 0.5 $\mathrm{mg}$ of atropine. At induction Propofol was administered with TCI through infusion pumps, the target plasma dose was $4 \mathrm{mcg} / \mathrm{ml}$ and was decreased to $3 \mathrm{mcg} / \mathrm{ml}$ during the operation. Remifentanil was administered as a continuous intravenous infusion, the dose was $0.25 \mu \mathrm{g} \mathrm{kg}-1 \mathrm{~min}-1$, and it was lowered to $0.15 \mu \mathrm{g} \mathrm{kg}-1$ min-1 starting surgery. This combination was regulated during surgery to reach a target arterial pressure equal to $25 \%$ of the baseline mean arterial pressure (MAP). After PN the MAP was gradually returned to baseline. 0.1-0.5 mg/kg cisatracurium besylate was given to facilitate orotracheal intubation, followed by the continuous application of 0.06-0.12 mg kg-1 h-1 cisatracurium via infusion pumps. The lungs were mechanically ventilated in a volume control mode with settings aimed at achieving normocapnia, reaching a tidal volume up to 8-10 $\mathrm{ml} / \mathrm{kg}$ and a respiratory frequency of $10-12$ breaths $/ \mathrm{min}$. Mechanical ventilation was initiated with a mixture of 50\% 02 and $50 \%$ air, and the inspired oxygen concentration was $40 \%$ during surgery. No patient received inotropes, vasopressors or methoclopramide during or after surgery. Monitoring included evaluation of cardiac hemodynamic parameters (electrocardiogram, heart rate, invasive blood pressure, systolic, diastolic, mean blood pressure [MAP]), respiratory parameters (pulseoximetry, end-tidal $\mathrm{CO} 2$, airway pressure). 


\section{Kidney Blood Pressure Research}

\section{Kidney Blood Press Res 2013;38:181-185}

\begin{tabular}{l|l}
\hline DOI: $10.1159 / 000355766$ & (C) 2014 S. Karger AG, Base
\end{tabular}

Published online: Aprit 08, 2014

www.karger.com/kbr
After surgery, the residual neuromuscular blockade was reversed with a mixture of atropine and neostigmine, only if deemed clinically necessary. Anesthetic agents were switched off, and $100 \% 02$ was given with $8 \mathrm{l} / \mathrm{min}$ fresh gas flow for $1 \mathrm{~min}$. Supplemental oxygen was not given postoperatively.

Hypothermic prevention during anesthesia was achieved by warm venous infusion (warmed serum), and a thermal blanket was applied to cover the upper part of the body. In addition, a warming forced-air blanket was used post-surgery.
Table 1.

\begin{tabular}{lc}
\hline & \\
\hline Liquids (ml) & $2020 \pm 343$ \\
Propofol (mg) total dose & $257 \pm 33$ \\
Remifentanil (ug) total dose & $417 \pm 34$ \\
\hline
\end{tabular}

Table 2.

\begin{tabular}{lcccc}
\hline Time & MAPmmHg & CF & Hct (\%) & Hb gr/dl \\
\hline T0 & $83 \pm 7$ & $72 \pm 9$ & $44.0 \pm 1.9$ & $13 \pm 0.65$ \\
T1 & $68 \pm 2$ & $70 \pm 6$ & $40.5 \pm 1.7$ & $12 \pm 0.75$ \\
T2 & $80 \pm 6$ & $71 \pm 7$ & $36 \pm 1.6$ & $12 \pm 0.70$ \\
T3 & $83 \pm 7$ & $72 \pm 9$ & $35 \pm 1.1$ & $11 \pm 0.60$ \\
\hline
\end{tabular}

\section{Results}

The zero ischemia procedure with $\mathrm{CH}$ was successfully completed in all patients. The mean age was $57.1 \pm 11.5 \mathrm{yr}$, body mass index (BMI) was $26.8 \pm 8.4 \mathrm{~kg} / \mathrm{m} 2$. The mean tumor size was $3.2 \mathrm{~cm}$ (ranging from 2 to 7), mean operative time was 100 minutes (ranging from 60 to 155), the mean duration of $\mathrm{CH}$ was 50 minutes (ranging from 46 to 54), mean blood loss was $200 \mathrm{ml}$ (ranging from 30 to 500) and median hospital stay was 3 days (range 3 to 8). Total amount of liquid infusion and anesthetic agents administered are reported in Table 1. Mean MAP, hematocrit and hemoglobin at T0, T1, T2 and T3, were reported in Table 2. Early postoperative complications were noted in 2 patients: retroperitoneal hematoma (Clavien grade 2), which were treated conservatively. No late postoperative complications occurred.

Variation of serum creatinine, blood urea nitrogen (BUN), eGFR, fractional sodium excretion (FSE), during the procedure and postoperatively are shown in Table 3. Acute renal failure was not observed in any of the patients. A significant variation of eGFR at T1, T2 and T3 was observed. No significant variation of BUN and FSE was detected. Comparing preoperative ERPF of the operated kidney with ERPF 3 months after surgery, it decreased by $2 \%$.

\section{Discussion}

WIT is associated with an increased risk of ARF in the postoperative period and new onset development of stage IV chronic kidney disease [7].

In a recent review, Simone et al. emphasized that, despite the increasing evidence of WIT as the only variable that the surgeon can significantly impact to minimize the loss of renal function after PN, MIPN is today conventionally performed after clamping hilar vessels (artery only or artery and vein) [8]. Therefore, efforts to limit or better eliminate ischemia time has been considered when performing PN.

Despite different definitions such as "off clamp", or "clamp-less" or "unclamped" PN, several Authors reported feasibility and effectiveness of MIPN without hilar clamp before 2011, when Gill et al. introduced the concept of "Zero Ischemia" MIPN [3].

In 2007 Gallucci et al. reported a preliminary experience on laparoscopic PN following superselective transarterial tumor embolization, highlighting feasibility and effectiveness of this procedure [9].

This technique provided efficient bleeding control and allowed surgeons to perform MIPN also in patients with cT1b renal tumors with moderate nephrometry score [5-10]. The same group demonstrated how a Zero Ischemia sutureless MIPN can be successfully 


\section{Kidney Blood Pressure Research}

performed in more than $95 \%$ of renal tumors with low nephrometry score [11]. In 2011 Gill et al. reported the feasibility of "Zero Ischemia" MIPN under $\mathrm{CH}$ without increased complication rate (one Clavien grade 1 , one Clavien grade 2 and one Clavien Grade 4a) and with negligible renal function loss.

Following steps of Gill et al. [3] we found $\mathrm{CH}$ to be an effective procedure to avoid hilar clamping during MIPN and able to guarantee optimal renal function preservation; in this series, median absolute and percent postoperative e-GFR decrease was $11.6 \mathrm{~mL} /$ min and $13.3 \%$, respectively [4].

Historically, yet in the first half of the twentieth century, $\mathrm{CH}$ emerged as a tool able to minimize intraoperative blood loss and the first controlled study was published in 1966 [12]. HA in patients scheduled for total hip arthroplasty had significantly lower intraoperative blood loss and operating time compared to the normotensive group. No significant differences were observed in mortality and morbidity in terms of cerebral, cardiovascular, renal, and hepatic function between the hypotensive and the normotensive groups [13].

More recently, the anatomic vascular microdissection (VMD) technique was described in a paper by Casey et al. from the same group (University of Southern California) comparing two series of MIPN performed with or without dissection of tumor specific tertiary or higher order renal arterial branches (Group 1=22 patients with VMD and Group 2=22 patients without VMD). Once confidence with superselective VMD was achieved satisfactorily, the authors no longer used CH anesthesia to perform MIPN [14].

In the present study we investigated renal function under long lasting $\mathrm{CH}$ during Zero Ischemia MIPN.

In our previous experience, according to steps described by Gill et al., HA started just before the incision of the renal parenchyma and lasted only the time necessary for tumor resection: median duration of $\mathrm{CH}$ with a median MAP of $65 \mathrm{~mm} \mathrm{Hg}$ (range: $55-70 \mathrm{~mm} \mathrm{Hg}$ ) was 14 min (range: $7-16 \mathrm{~min}$ ) [4].

In this study the mean duration of $\mathrm{CH}$ was $50 \pm 4$ minutes. Despite a significant variation in serum creatinine and eGFR was observed, none of the patients in this series has experienced either acute renal failure or temporary dialysis.

Three months after surgery (T4) eGFR was comparable with the preoperative levels (table 3). As such, we may conclude that if a patient had good renal function preoperatively, zero ischemia RPN under $\mathrm{CH}$ does not cause new-onset CKD, and the renal function undergoes a temporary reduction that tends to recover in less than a month. In our experience $\mathrm{CH}$ was well tolerated in all patients, and there were no adverse effects. Further studies are needed to validate our results. Limitations of this study include the prospective cohort study, the non-randomized study design, and the acquisition of operative data from a single institution by a single experienced surgeon. 


\section{Kidney \\ Blood Pressure Research}

Forastiere/Claroni/Sofra et al.: Renal Function After Controlled Hypotension

\section{Conclusions}

Analysis of surgical and renal functional outcomes of RPN under $\mathrm{CH}$ confirms its safety and efficacy in selected cases. After a phase of initial alteration of renal function, this is completely recovered to 30 days. These findings reaffirm that RPN under $\mathrm{CH}$ represents a reliable NSS procedure, offering effective renal function preservation avoiding hilar clamping.

\section{Conflict of Interests}

The authors declare that they do not have any conflicts.

\section{References}

-1 Ljungberg B, Cowan NC, Hanbury DC, Hora M, Kuczyk MA, Merseburger AS, Patard JJ, Mulders PF, Sinescu IC: EAU guidelines on renal cell carcinoma: the 2010 update. Eur Urol 2010;58:398-406.

2 Touijer K, Guillonneau B: Advances in laparoscopic partial nephrectomy. Curr Opin Urol 2004;14:235-237.

3 Gill IS, Eisenberg MS, Aron M, Berger A, Ukimura O, Patil MB, Campese V, Thangathurai D, Desai MM: "Zero ischemia" partial nephrectomy: novel laparoscopic and robotic technique. Eur Urol 2011;59:128-134.

4 Papalia R, Simone G, Ferriero M, Costantini M, Guaglianone S, Forastiere E, Gallucci M: Laparoscopic and robotic partial nephrectomy with controlled hypotensive anesthesia to avoid hilar clamping: feasibility, safety and perioperative functional outcomes. J Urol 2012;187:1190.

-5 Simone G, Papalia R, Guaglianone S, Forestiere E, Gallucci M: Preoperative superselective transarterial embolization in laparoscopic partial nephrectomy: technique, oncologic and functional outcomes. J Endourol 2009;23:1473.

6 Levey AS, Stevens LA, Schmid CH, Zhang YL, Castro AF 3rd, Feldman HI, Kusek JW, Eggers P, Van Lente F, Greene T, Coresh J: CKD-EPI (Chronic Kidney Disease Epidemiology Collaboration). A New Equation to Estimate Glomerular Filtration Rate. Ann Intern Med 2009;150:604-612.

7 Thompson RH, Lane BR, Lohse CM, Leibovich BC, Fergany A, Frank I, Gill IS, Blute ML, Campbell SC: Every minute counts when the renal hilum is clamped during partial nephrectomy. Eur Urol 2010;58:340.

-8 Simone G, Ferriero M, Papalia R, Costantini M, Guaglianone S, Gallucci M: Zero-ischemia minimally invasive partial nephrectomy. Curr Urol Rep 2013;14:465-470.

-9 Gallucci M, Guaglianone S, Carpanese L, Papalia R, Simone G, Forestiere E, Leonardo C: Superselective embolization as first step of laparoscopic partial nephrectomy. Urology 2007;69:642-646.

10 Simone G, Papalia R, Guaglianone S, Carpanese L, Gallucci M: Zero ischemia laparoscopic partial nephrectomy after superselective transarterial tumor embolization for tumors with moderate nephrometry score: long-term results of a single-center experience. J Endourol 2011;25:1443-1446.

11 Simone G, Papalia R, Guaglianone S, Gallucci M: 'Zero ischaemia' sutureless laparoscopic partial nephrectomy for renal tumours with a low nephrometry score. BJU Int 2011;110:124-130.

12 Eckenhoff JE, Rich JC: Clinical experiences with deliberate hypotension. Anesth Analg 1966;45:21.

13 Thompson GE, Miller RD, Stevens WC, Murray WR: Hypotensive anesthesia for total hip arthroplasty: a study of blood loss and organ function (brain, heart, liver, and kidney). Anesthesiology 1978;48:91.

14 Ng CK, Gill IS, Patil MB, Hung AJ, Berger AK, de Castro Abreu AL, Nakamoto M, Eisenberg MS, Ukimura O, Thangathurai D, Aron M, Desai MM: Anatomic renal artery branch microdissection to facilitate zeroischemia partial nephrectomy. Eur Urol 2012;61:67-74. 